\title{
How to tell if a group law entails virtual nilpotence: postscript
}

\author{
R. G. Burns, O. N. Macedońska, and Yuri Medvedev
}

Department of Mathematics and Statistics, York University, Toronto, On M3J 1P3, Canada

E-mail: rburns@mathstat.yorku.ca

Institute of Mathematics, Silesian University of Technology, Kaszubska 23, 44-100 Gliwice, Poland

E-mail: olga.macedonska@polsl.pl

EBS, Bank of Montreal, 3300 Bloor Street West, 14th Floor, West Tower, Toronto, On M8X 2X3, Canada

E-mail: yuri.medvedev@bmo.com

\begin{abstract}
It is known that for any finitely generated group $G$ from the large class of "locally graded" groups, satisfaction of an Engel or positive law forces $G$ to be virtually nilpotent. In [2] Sarah Black gives a sufficient condition for an arbitrary 2-variable law $w(x, y) \equiv 1$ to imply virtual nilpotence - though only for finitely generated residually finite groups. We show how the Dichotomy Theorem from [4] for arbitrary words $w\left(x_{1}, \ldots, x_{n}\right)$, encompasses Black's condition, extending it to the $n$ variable case and a certain large class $\mathcal{S}$ (however still falling short of the class of locally graded groups). We infer in particular that her condition is also necessary. We also deduce a simplified version of an algorithm of Qianlu Li [8, 9] for deciding whether or not a given law $w\left(x_{1}, \ldots, x_{n}\right) \equiv 1$ satisfies the extended version of Black's criterion.

Key words: word, group law, virtual nilpotence, wreath product, variety
\end{abstract}

2000 MR Subject Classification: 20E10, 20E22, 20F10 
There are two kinds of group law that have received a great deal of attention from group-theorists over a long period, namely the

$$
n-\text { Engel law }[x, \underbrace{y, \ldots, y}_{n}] \equiv 1, n=1,2, \ldots,
$$

(where, as usual, $[x, y]:=x^{-1} y^{-1} x y,[x, y, y]:=[[x, y], y]$, etc.), and

$$
\text { positive (or monoidal) laws } u\left(x_{1}, x_{2}, \ldots\right) \equiv v\left(x_{1}, x_{2}, \ldots\right) \text {, }
$$

where $u, v$ are distinct words in the free group $F\left(x_{1}, x_{2}, \ldots\right)$ with free basis $x_{1}, x_{2}, \ldots$, involving only nonnegative powers of $x_{1}, x_{2}, \ldots$

Each of these kinds of law is related to the class- $n$ nilpotent law

$$
[x, \underbrace{y, z, \ldots}_{n}] \equiv 1, n=1,2, \ldots
$$

This is obvious in the case of the $n$-Engel law, which is a specialization of the $n$-nilpotent law. On the other hand satisfaction of a positive law may be considered as a generalization of $n$-nilpotence, in view of the result of $\mathrm{Mal}^{\prime} \mathrm{cev}$ [11] that the $n$-nilpotent law is equivalent to a certain positive law. (This was independently discovered by B. H. Neumann and Tekla Taylor [12] somewhat later.) Thus in each case the following question naturally arose:

Does each of these two kinds of law imply (at least local) nilpotence?

In the case of the $n$-Engel law the answer is unknown. (The best result applying to all groups is that of Havas and Vaughan-Lee [6] that every 4Engel group must be locally nilpotent. For general $n$, on the other hand, the best result to date is that of Kim and Rhemtulla [7] asserting that a "locally graded" $n$-Engel group must be locally nilpotent. A locally graded group is one in which every nontrivial finitely generated subgroup has a proper subgroup of finite index. A somewhat stronger version of the result of Kim and Rhemtulla can be found in [4].)

Turning to positive laws, we observe first that the most we can hope for is that a finitely generated group satisfying a positive law should be nilpotentby-(finite exponent). An example dashing this hope is given in [14]. Here the best positive result so far - the last of a long line of such results - would seem to be the following one.

TheOREM [3,4]. For any positive law $u \equiv v$ the locally graded groups satisfying that law are all (nilpotent of class d)-by-(locally finite of exponent e) for some d,e depending only on $u, v$. 
The following question then naturally arises:

What do the Engel laws and positive laws have in common that forces finitely generated locally graded groups satisfying them to be nilpotent-byfinite?

The following partial answer, in the 2-variable case, was given by Sarah Black. We reformulate her result in terms of the restricted wreath product $W:=C$ < $C$ where $C$ is infinite cyclic. We write $c$ for a generator of a coordinate subgroup, and $t$ for a generator of the top group; thus $W$ is generated by $c$ and $t$, in terms of which it is defined by the relations

$$
\left[c, c^{t^{k}}\right]=1, k \in \mathbb{Z}
$$

where we are using the notation $a^{b}:=b^{-1} a b$ for any elements $a, b$ of a group.

Sarah BlacK's Theorem [2]. Let $F=F(x, y)$ denote the free group freely generated by $x, y$, and let $w(x, y)$ be any word from $F^{\prime}:=[F, F]$, the commutator subgroup of $F$, not contained in $F^{\prime \prime}=\left[F^{\prime}, F^{\prime}\right]$, with the following property: the law $w(x, y) \equiv 1$ has a consequence $\hat{w}(x, y) \equiv 1$ (i.e., $\hat{w}(x, y)$ is an element of the verbal subgroup of $F$ generated by $w(x, y))$ such that $\hat{w}(x, y) \notin F^{\prime \prime}$, and if $\hat{w}(c, t) \in W$ has the canonical form

$$
\left(c^{m_{1}}\right)^{t^{k_{1}}} \cdots\left(c^{m_{r}}\right)^{t^{k_{r}}}, r>0, m_{1}, m_{2}, \ldots, m_{r} \neq 0, k_{1}<k_{2}<\cdots<k_{r},
$$

and $\hat{w}(t, c)$ the form

$$
\left(c^{n_{1}}\right)^{t^{l_{1}}} \cdots\left(c^{n_{s}}\right)^{t^{l_{s}}}, s>0, n_{1}, \ldots, n_{s} \neq 0, l_{1}<l_{2}<\cdots<l_{s},
$$

then

$$
\delta(\hat{w}):=\operatorname{gcd}\left(m_{1}, \ldots, m_{r} ; n_{1}, \ldots, n_{s}\right)=1 .
$$

Then every residually finite group satisfying the law $w(x, y) \equiv 1$ is (nilpotent of class d)-by-(locally finite of exponent e) where $d$ and e depend only on the word $w(x, y)$.

Remarks. 1. If $w(x, y) \notin F^{\prime}$, then $w(x, y) \equiv 1$ has a consequence of the form $x^{n} \equiv 1$ for some $n>1$, so that the conclusion of the theorem holds also in this case.

2. In [2] this theorem is formulated without explicit mention of the wreath product $W$, but rather in terms of elements of the free group $F(x, y)$, expressed modulo the kernels of the two epimorphisms $F(x, y) \rightarrow W$ defined by $x \rightarrow c, y \rightarrow t$, and $x \rightarrow t, y \rightarrow c$. 
3. Write $W_{p}:=C_{p} \imath C$, where $C_{p}$ is the cyclic group of prime order $p$, and denote by $c_{p}$ a generator of any coordinate subgroup of $W_{p}$, and by $t$, as before, a generator of the top group $C$. Then $W_{p}$ is generated by $c_{p}$ and $t$ with the defining relations

$$
c_{p}^{p}=1, \quad\left[c_{p}, c_{p}^{t^{k}}\right]=1, \quad k \in \mathbb{Z} .
$$

The assumption (2) concerning $w(x, y)$ in Black's theorem may then be reformulated in terms of the groups $W_{p}$ as follows:

There should exist a consequence $\hat{w}(x, y) \equiv 1$ of $w(x, y) \equiv 1$ such that for all primes $p$ either $\hat{w}\left(c_{p}, t\right) \neq 1$ or $\hat{w}\left(t, c_{p}\right) \neq 1$ in $W_{p}$.

Motivated by Sarah Black's theorem we (chiefly Medvedev) were able to prove the following precise dichotomy for words, significantly extending her result. We first define the class $\mathcal{S}$ of all groups obtainable from the class of groups that are soluble-by-(locally finite of finite exponent) by closing under the operators $L$ and $R$, where $L \mathcal{X}$ denotes the class of groups locally in the group-theoretic class $\mathcal{X}$, and $R \mathcal{X}$ the class of groups residually in $\mathcal{X}$. Note that the class $\mathcal{S}$ includes all residually finite and locally finite groups, and is a proper subclass of the class of locally graded groups.

Dichotomy Theorem [4]. Let $w\left(x_{1}, x_{2}, \ldots\right)$ be any word in $F\left(x_{1}, x_{2}, \ldots\right)$. Exactly one of the following two possibilities occurs:

(i) Every group in the class $\mathcal{S}$ satisfying the law $w \equiv 1$ is (nilpotent of class d)-by-(locally finite of exponent $e$ ), where $d$ and $e$ depend only on the word $w$.

(ii) There exists a prime $p$ such that $w \equiv 1$ is a law in $W_{p}$.

REMARKs (continued). 4. The proof of this result, like that of Sarah Black's theorem, depends heavily on results of Aner Shalev, E. I. Zelmanov, A. Lubotzky and A. Mann, and, in the last step in establishing the dichotomy, J. R. J. Groves. (Precise references may be found in [4].)

5. Since $W_{p}$ generates the product variety $\mathfrak{A}_{p} \mathfrak{A}$ of the variety of all abelian groups of exponent $p$ by the variety of all abelian groups (see e.g. [13, Corollary 22.44]), it follows from this dichotomy that the question as to whether a given word $w$ "spells virtual nilpotence" is equivalent to that asking if the law $w \equiv 1$ does not follow from a law $[x, y]^{p} \equiv 1$ and the metabelian law $\left[\left[x_{1}, x_{2}\right],\left[x_{3}, x_{4}\right]\right] \equiv 1$.

6. For some laws $w\left(x_{1}, x_{2}, \ldots\right) \equiv 1$-for instance $n$-Engel laws and positive laws - the class $\mathcal{S}$ in alternative (i) of the Dichotomy Theorem can be replaced by the significantly larger class of locally graded groups. If the law 
$w \equiv 1$ is such that groups $G$ satisfying it are uniformly restrained, i.e., there exists a $k$ such that for all $a, b \in G$ one has that the subgroup generated by all conjugates $a^{b^{l}}, l \in \mathbb{Z}$, can be generated by $\leq k$ elements, then this replacement may be made $[3,4]$. Groups satisfying an Engel or positive law are uniformly restrained. However it remains unknown whether in general the Dichotomy Theorem remains valid if $\mathcal{S}$ is replaced by the class of locally graded groups. (In [10] it is shown that the Dichotomy Theorem holds for locally graded groups if and only if every locally graded group containing a free non-abelian semigroup generates a variety containing $\mathfrak{A}_{p} \mathfrak{A}$ for some prime $p$. It is also noted in [10] that the Dichotomy Theorem does not hold for all groups, this following, for instance, from the construction by Ol'shanskiu [15] of a law $w \equiv 1$ defining a non-abelian variety all of whose metabelian (and finite) groups are abelian.)

Sarah Black's sufficient condition is immediate from this Dichotomy Theorem (see Remark 3 above.) That her condition is also necessary also follows readily:

Corollary 1. The sufficient condition of Sarah Black's theorem for a law $w(x, y) \equiv 1$ with $w \in F^{\prime} \backslash\left[F^{\prime}, F^{\prime}\right]$ to entail virtual nilpotence in any residually finite group (in fact in any group in the class $\mathcal{S}$ ) satisfying it, is also necessary.

Proof. For suppose the law $w(x, y) \equiv 1$ is such that none of its consequences $\hat{w}(x, y) \equiv 1$ satisfies $(2)$, i.e, $\delta(\hat{w})>1$ for all consequences $\hat{w}(x, y) \equiv 1$ with $\hat{w} \notin\left[F^{\prime}, F^{\prime}\right]$. We first show that there exists a prime $p$ dividing every $\delta(\hat{w})$. For, otherwise there would exist finitely many $\hat{w}$, say

$$
\hat{w}_{1}, \ldots, \hat{w}_{k}
$$

with $\operatorname{gcd}\left(\delta\left(\hat{w}_{1}\right), \delta\left(\hat{w}_{2}\right), \ldots, \delta\left(\hat{w}_{k}\right)\right)=1$. However then for suitably spaced integers $\sigma_{2}, \ldots, \sigma_{k}$ we would have

$$
\delta\left(\hat{w}_{1}\left(\hat{w}_{2}\right)^{t^{\sigma_{2}}} \cdots\left(\hat{w}_{k}\right)^{t^{\sigma_{k}}}\right)=1
$$

a contradiction. (The integers $\sigma_{2}, \ldots, \sigma_{k}$ are chosen so that there is no overlap in the (finite) supports of any two of $\hat{w}_{1},\left(\hat{w}_{2}\right)^{t^{\sigma_{2}}}, \ldots,\left(\hat{w}_{k}\right)^{t^{\sigma}}$, considered as functions $C \rightarrow C$.)

Thus there is a prime $p$ dividing $\delta(\hat{w})$ for all consequences $\hat{w} \equiv 1$ of $w \equiv 1$ $\left(\hat{w} \notin\left[F^{\prime}, F^{\prime}\right]\right)$. Hence by Remark 3 all such $\hat{w}(x, y)$ satisfy $\hat{w}\left(c_{p}, t\right)=1$ (and $\left.\hat{w}\left(t, c_{p}\right)=1\right)$. Let $g_{1}, g_{2}$ be arbitrary elements of $W_{p}$, and let $\gamma_{1}(x, y), \gamma_{2}(x, y)$ 
be words from $F(x, y)$ such that $g_{1}=\gamma_{1}\left(c_{p}, t\right)$ and $g_{2}=\gamma_{2}\left(c_{p}, t\right)$. Write $\hat{w}_{1}(x, y):=w\left(\gamma_{1}(x, y), \gamma_{2}(x, y)\right)$. Then $w\left(g_{1}, g_{2}\right)=\hat{w}_{1}\left(c_{p}, t\right)$. However since $\hat{w}_{1} \equiv 1$ is a consequence of $w \equiv 1$, it follows from what we have already proved that $\hat{w}_{1}\left(c_{p}, t\right)=1$, whence $w\left(g_{1}, g_{2}\right)=1$, and since $g_{1}, g_{2}$ are arbitrary elements of $W_{p}$, we conclude that $w \equiv 1$ is a law in $W_{p}$, so that the second alternative of the Dichotomy Theorem holds for $w$, i.e., the word $w$ does not "spell virtual nilpotence".

REMARK 7. The referee has pointed out that Qianlu Li [9, Theorem 23] has shown that if a word "spells virtual nilpotence," then it has an "efficient result", i.e., a consequence in a certain special sense, satisfying Sarah Black's criterion (2). The above proof seems rather simpler than Li's, although on the face of it not quite so general. We note also the result of G. Endimioni [5, Lemma 4], somewhat resembling our Corollary 1.

ExAmples. Among her examples Sarah Black considers the particular law $w=\left[x^{2}, y^{2}, x^{2}\right] \equiv 1$, deducing that, although it itself does not satisfy the condition (2), some consequence does. Now it is easy to check that in fact no law of the form $\left[x^{k}, y^{l}, x^{m}\right] \equiv 1$ with $k, l, m \neq 0$, holds in any $W_{p}$, so that by the Dichotomy Theorem any group from $\mathcal{S}$ satisfying such a law must be indeed nilpotent-by-(locally finite of finite exponent). On the other hand any law $w \equiv 1$ with $w$ of the form $w=v^{k}$, where $k>0$ and $v=v\left(x_{1}, x_{2}, \ldots\right)$ is in the commutator subgroup of $F\left(x_{1}, x_{2}, \ldots\right)$, is clearly a law in $W_{p}$ for each $p$ dividing $k$, so does not "spell 'almost nilpotent'."

There remains the problem of finding an explicit algorithm for deciding, for any given word $w\left(x_{1}, x_{2}, \ldots\right)$, which side of the above dichotomy it falls on. Such an algorithm has been given by Qianlu Li in $[8,9]$, which, although not formulated explicitly in terms of the wreath product $W:=C \imath C$, again begs to be so formulated, in our opinion.

We now describe such an algorithm, equivalent to Qianlu Li's in its essentials, but couched in terms of the wreath product $W$, and somewhat simplified.

Thus let $w=w\left(x_{1}, x_{2}, \ldots, x_{n}\right)$ be any word in the free group $F\left(x_{1}, x_{2}, \ldots\right)$. We seek a procedure for deciding whether or not $w \equiv 1$ is a law in $W_{p}:=C_{p} \imath C$ for some prime $p$.

If any generator $x_{i}$ occurs in $w$ with exponent sum $k \neq 0$, then, essentially for the reasons given in Remark 1, w satisfies the alternative (i) of the Dichotomy Theorem. 
On the other hand if $w$ belongs to the commutator subgroup of $F\left(x_{1}, x_{2}, \ldots\right)$, then $w \equiv 1$ is a law in $W_{p}$ if and only if, on performing any substitution $x_{i} \rightarrow b_{i} t^{k_{i}}, i=1,2, \ldots$, where $t$ is, as before, a generator of the top group of $W:=C \imath C$, and $b_{i}$ is an element of the base group, we always obtain a base element with canonical form

$$
\left(c^{i_{1}}\right)^{t^{j_{1}}}\left(c^{i_{2}}\right)^{t^{j_{2}}} \cdots\left(c^{i_{r}}\right)^{t^{j_{r}}}, r \geq 0, i_{1}, i_{2}, \ldots, i_{r} \neq 0, j_{1}<j_{2}<\cdots<j_{r},
$$

with $p$ dividing each of $i_{1}, i_{2}, \ldots, i_{r}$. It is not difficult to see using the relations (1) and the present assumption that each $x_{i}$ occurs in $w$ with exponent sum zero, that for any $i$, if $b_{i}=b_{i}^{\prime} b_{i}^{\prime \prime}$ is any factorization of $b_{i}$ as a product of two elements of the base group of $W$, then

$$
\begin{aligned}
& w\left(b_{1} t^{k_{1}}, b_{2} t^{k_{2}}, \ldots, b_{i}^{\prime} b_{i}^{\prime \prime} t^{k_{i}}, \ldots, b_{n} t^{k_{n}}\right)= \\
& \quad w\left(b_{1} t^{k_{1}}, b_{2} t^{k_{2}}, \ldots, b_{i}^{\prime} t^{k_{i}}, \ldots, b_{n} t^{k_{n}}\right) w\left(t^{k_{1}}, t^{k_{2}}, \ldots, b_{i}^{\prime \prime} t^{k_{i}}, \ldots, t^{k_{n}}\right) .
\end{aligned}
$$

(This can most easily be seen as follows. The base group of $W$ may be regarded as a (cyclic) $\mathbb{Z}[C]$-module, in terms of which $t^{k} c^{l} t^{-k}$ becomes $l t^{k} c$ for any integers $k, l$. The above equation then derives from the following one, where the $\rho_{s}$ are arbitrary elements of the group $\operatorname{ring} \mathbb{Z}[C]$ and the $\beta$ s module elements, i.e., elements of the base group of $W$ written additively:

$$
\begin{aligned}
\rho_{1} \beta_{1}+\rho_{2} \beta_{2}+\cdots+\rho_{i}\left(\beta_{i}^{\prime}+\beta_{i}^{\prime \prime}\right)+\cdots+\rho_{n} \beta_{n} & = \\
& \left(\rho_{1} \beta_{1}+\rho_{2} \beta_{2}+\cdots+\rho_{i} \beta_{i}^{\prime}+\cdots+\rho_{n} \beta_{n}\right)+\rho_{i} \beta_{i}^{\prime \prime},
\end{aligned}
$$

- a trivial consequence of the defining properties of a module.)

Hence we obtain the following result:

Corollary 2. Given any word $w\left(x_{1}, x_{2}, \ldots, x_{n}\right)$ in the commutator subgroup of the free group $F\left(x_{1}, x_{2}, \ldots\right)$, the law $w \equiv 1$ holds in $W_{p}$ if and only if for all integers $k_{1}, k_{2}, \ldots, k_{n}$, each of the $n$ elements

$$
w\left(c t^{k_{1}}, t^{k_{2}}, \ldots, t^{k_{n}}\right), w\left(t^{k_{1}}, c t^{k_{2}}, \ldots, t^{k_{n}}\right), \ldots, w\left(t^{k_{1}}, t^{k_{2}}, \ldots, c t^{k_{n}}\right)
$$

of $W$ has canonical form (3) with $p$ dividing each of $i_{1}, i_{2}, \ldots, i_{r}$.

ExAmple. In [9] the example $w=x_{1}^{2} x_{2}^{-1} x_{3}^{3} x_{2} x_{3}^{-1} x_{1}^{-2} x_{3}^{-2}$ is considered. Since this is in the commutator subgroup of $F\left(x_{1}, x_{2}, \ldots\right)$, we can apply Corollary 2 to see if it satisfies the alternative (ii) of the Dichotomy Theorem. 
Thus we need to consider for each triple of integers $k_{1}, k_{2}, k_{3}$ the following three elements of the wreath product $W$ :

$$
\begin{gathered}
\left(c t^{k_{1}}\right)^{2} t^{-k_{2}} t^{3 k_{3}} t^{k_{2}} t^{-k_{3}}\left(c t^{k_{1}}\right)^{-2} t^{-2 k_{3}}, \\
t^{2 k_{1}}\left(c t^{k_{2}}\right)^{-1} t^{3 k_{3}}\left(c t^{k_{2}}\right) t^{-k_{3}} t^{-2 k_{1}} t^{-2 k_{3}}, \\
t^{2 k_{1}} t^{-k_{2}}\left(c t^{k_{3}}\right)^{3} t^{k_{2}}\left(c t^{k_{3}}\right)^{-1} t^{-2 k_{1}}\left(c t^{k_{3}}\right)^{-2} .
\end{gathered}
$$

The first of these has canonical form

$$
c c^{t^{-k_{1}}}\left(c^{-1}\right)^{t^{-2 k_{3}-k_{1}}}\left(c^{-1}\right)^{t^{-2 k_{3}}}
$$

and since for suitable choice of the integers $k_{1}, k_{2}, k_{3}$, the four exponents $0,-k_{1},-2 k_{3}-k_{1},-2 k_{3}$ will be distinct, with associated powers of $c$ equal to $1,1,-1,-1$ respectively, it is immediate from Corollary 2 (there is no need to examine the other two elements of $W$ ) that this word does not satisfy the alternative (ii) of the dichotomy. Hence it must satisfy the alternative (i), i.e., any group in the class $\mathcal{S}$ satisfying the corresponding law $w \equiv 1$ must be nilpotent-by-(locally finite of finite exponent).

\section{REFERENCES}

[1] Beata Bajorska, 'On the smallest class containing all finite and all soluble groups, locally and residually closed,' Publicationes Mathematicae, Debrecen (to appear).

[2] Sarah Black, 'Which words spell "almost nilpotent"?,' J. Algebra 221(1999), 475-496.

[3] R. G. Burns, O. N. Macedońska and Y. Medvedev, 'Groups satisfying semigroup laws, and nilpotent-by-Burnside varieties', J. Algebra 195(1997), 510-525.

[4] R. G. Burns and Yuri Medvedev, 'Group laws implying virtual nilpotence,' J. Aust. Math. Soc. 74(2003), 295-312.

[5] G. Endimioni, 'On the locally finite $p$-groups in certain varieties of groups,' Quart. J. Math. Oxford Series 48(1997), 169-178.

[6] George Havas and M. Vaughan-Lee, '4-Engel groups are locally nilpotent,' (preprint).

[7] Y. K. Kim and A. H. Rhemtulla, 'Weak maximality conditions and polycyclic groups,' Proc. Amer. Math. Soc. 123(1995), 711-714. 
[8] Qianlu Li, 'Which words have an efficient result?,' Commun. Algebra 32(2004), 2527-2545.

[9] Qianlu Li, 'Words and almost nilpotent varieties of groups,' Commun. Algebra 33(2005), 3569-3582.

[10] O. N. Macedońska, (preprint)

[11] A. I. Mal'cev, 'Nilpotent semigroups,' Uchen. Zap. Ivanovsk. Ped. Inst. 4 (1953), 107-111.

[12] B. H. Neumann and Tekla Taylor, 'Subsemigroups of nilpotent groups,' Proc. Roy. Soc. Ser. A 274(1963), 1-4.

[13] H. Neumann, Varieties of groups (Springer, Berlin, 1967).

[14] A. Yu. Ol'shanskiı̌ and A. Storozhev, 'A group variety generated by a semigroup law,' J. Aust. Math. Soc. Ser. A 60(1996), 255-259.

[15] A. Yu. Ol'shanskiü, Geometry of defining relations in groups (Kluwer Academic Publishers, Dordrecht, 1991.) 